\title{
Different distribution of abnormal endocardial electrograms within the right atrium in patients with sick sinus syndrome
}

Third Department of Internal Medicine, Nagasaki University School of Medicine, Nagasaki, Japan O A Centurion M Fukatani A Konoe M Tanigawa

A Shimizu

S Isomoto

M Kaibara

K Hashiba

Correspondence to: Dr Osmar Antonio Centurion, Third Department of Internal Medicine, Nagasaki University School of Medicine, 7-1 Sakamotomachi, Nagasaki, 852 Japan Accepted for publication 9 June 1992.

Osmar Antonio Centurion, Masahiko Fukatani, Atsushi Konoe, Muneo Tanigawa, Akihiko Shimizu, Shojiro Isomoto, Muneshige Kaibara, Kunitake Hashiba

\begin{abstract}
Background-Prolonged and fractionated right atrial endocardial electrograms are characteristic of paroxysmal atrial fibrillation (idiopathic or associated with sick sinus syndrome). The distribution of these abnormal atrial electrograms within the right atrium and the way it is related to the likelihood that patients with sick sinus syndrome will develop paroxysmal atrial fibrillation was studied.
\end{abstract}

Methods-Endocardial catheter mapping of the right atrium during sinus rhythm was performed in 41 control patients with normal sinus node function and without paroxysmal atrial fibrillation, in 33 patients with sick sinus syndrome but without tachycardia, and in 27 patients with sick sinus syndrome and paroxysmal atrial fibrillation (group 3 ). The bipolar electrograms were recorded at 12 sites in the right atrium and an abnormal atrial electrogram was defined as lasting $\geqslant 100 \mathrm{~ms}$ and/or showing eight or more fragmented deflections.

Results-1195 atrial endocardial electrograms were assessed and quantitatively measured. In patients with sick sinus syndrome and paroxysmal atrial fibrillation $54 \%$ of the abnormal atrial electrograms were recorded from the high right atrium, $28 \%$ from the mid right atrium, and $18 \%$ from the low right atrium. However, in patients with sick sinus syndrome without tachycardia $78 \%$ of the abnormal atrial electrograms were recorded from the high right atrium and $22 \%$ from the mid right atrium. No abnormal electrograms were recorded from the low right atrium.

Conclusions-In patients with sick sinus syndrome without tachycardia abnormal atrial electrograms generally came from the high right atrium but in patients with sick sinus syndrome and paroxysmal atrial fibrillation abnormal atrial electrograms were more widely distributed in the right atrium. In patients with sick sinus syndrome the greater the extent of the compromised atrial muscle, the greater the likelihood that paroxysmal atrial fibrillation will develop.

(Br Heart J 1992;68:596-600)
Fragmented and continuous ventricular electrical activity was recorded from infarcted ventricular muscle in canine hearts ${ }^{12}$ and in patients with ischaemic heart disease. ${ }^{3-5}$ The electrograms are fractionated because muscle fibers are separated by scar tissue, which causes fragmented, slow, and anisotropic conduction. ${ }^{6}$

With intra-atrial catheter mapping we showed that a prolonged and fractionated atrial electrogram is characteristic of paroxysmal atrial fibrillation (either idiopathic or associated with sick sinus syndrome). However, the sites in the right atrium where abnormal atrial electrograms are generated in patients with sick sinus syndrome have not been described. About $15-40 \%$ of patients with sick sinus syndrome develop paroxysmal atrial fibrillation ${ }^{89}$ while the remainder do not. We wondered whether this reflected a difference in the extent and distribution of abnormal atrial electrograms in patients with sick sinus syndrome. We designed a study $(a)$ to determine the sites in the right atrium of patients with sick sinus syndrome at which electrical activity is likely to be abnormal and (b) to find a relation between the distribution of abnormal atrial electrograms and the likelihood that paroxysmal atrial fibrillation will develop in patients with sick sinus syndrome.

\section{PATIENTS}

One hundred and one patients referred to the Nagasaki University Hospital for electrophysiological evaluation of their arrhythmias underwent right atrial endocardial mapping in sinus rhythm during investigation. No patients with hypothyroidysm, congestive heart failure, organic heart disease, or cerebrovascular accidents were admitted to the study. None of the patients had had a recent myocardial infarction or operation. Neither drug treatment nor serum electrolyte disturbances were found to be related to the genesis of the sinus node dysfunction in our study patients. No patient in whom echocardiography showed atrial enlargement was included in this investigation.

We studied three groups of patients. Group 1 was 41 patients ( 19 women and 22 men) with normal sinus node function and without paroxysmal atrial fibrillation (age 41-83 years, mean (SD) $64.5(10 \cdot 5)$ ). Electrophysiological study was performed because of atrioventricular nodal reentrant tachycardia in 20 patients, advanced or complete atrioventricular block in 19 patients, and ventricular tachycardia in two patients. Because of their known predisposition 
to develop paroxysmal atrial fibrillation, patients with the Wolff-Parkinson-White syndrome $\mathrm{e}^{10-12}$ were not included in this group. The sinus node recovery time ranged from 0.72 to $1.34 \mathrm{~s}$ (mean (SD) $1.07(0.15) \mathrm{s})$. Group 2 consisted of 33 patients ( 17 women and 16 men) with sick sinus syndrome but without tachycardia (age 42-81 years, mean (SD) $63 \cdot 7(9 \cdot 3)$ ). Eleven patients had persistent and unexplained sinus bradycardia ( $<50$ beats $/ \mathrm{min}$ ) on the resting electrocardiogram, ${ }^{13}$ with fewer than 70 thousand beats per 24 hours on ambulatory Holter monitor recordings. ${ }^{14}$ The other 22 patients had documented episodes of sinoatrial block or sinus arrest with atrioventricular junctional or ventricular escape depolarisation. ${ }^{1516}$ The sinus node recovery time ranged from $1 \cdot 15$ to $6.88 \mathrm{~s}$ (mean (SD) $2.93(1.9) \mathrm{s}$ ). There was no clinical evidence of paroxysmal atrial fibrillation in any of these patients. Care was taken to avoid false diagnosis of sinoatrial block in the presence of premature non-conducted atrial depolarisation. Group 3 comprised 27 patients (18 women and nine men) (age 49-81 years, mean (SD) $66.7(8.9)$ ) with both sick sinus syndrome and paroxysmal atrial fibrillation, that is, the so-called bradycardia-tachycardia syndrome. All patients in this group had confirmed paroxysmal atrial fibrillation. The sinus node recovery time ranged from 1.20 to $9.28 \mathrm{~s}$ (mean (SD) $4 \cdot 23(2 \cdot 2) \mathrm{s}$ ). Sinus node dysfunction in the patients in groups 2 and 3 and the paroxysmal atrial fibrillation of the patients in group 3 were confirmed by conventional electrocardiography, bedside electrocardiographic monitoring, and/or at least two 24 hour ambulatory electrocardiographic Holter recordings. The sinus node recovery time was measured in all patients after atrial overdrive pacing ${ }^{17} 18$ at rates of $70-210 / \mathrm{min}$ for a minute at each level. There was no significant age difference among patients in the three groups.

\section{ELECTROPHYSIOLOGICAL STUDY}

All patients were studied in the postabsorptive, non-sedated state after they had given infor- med consent. Electrophysiological evaluation was performed after approval of the study plan by the regional ethics committee. All drugs were stopped at least 72 hours before the procedure. Two bipolar catheter electrodes (No 6F USCI) were inserted percutaneously into the femoral and subclavian veins and were advanced into the right atrium under fluoroscopic guidance. The distance between the electrodes was $10 \mathrm{~mm}$ and the electrode ring was $2 \mathrm{~mm}$ in diameter.

All the right atrial endocardial electrograms were recorded during sinus rhythm by bipolar electrodes at a fixed gain setting, accompanied by a $0.2 \mathrm{mV}=3 \mathrm{~mm}$ calibration signal, and were filtered at $50-1000 \mathrm{~Hz}$. The baseline of recordings was stable for each patient. The atrial endocardial electrograms together with the monophasic action potential and three surface electrocardiograms (I, aVF, and V1) were displayed on a multichannel oscilloscope (Fukuda-Denshi polygraph MIC-8800T) and simultaneously recorded at a paper speed of $100 \mathrm{~mm} / \mathrm{s}$ on a 12 channel, ink jet recorder (Siemens-Elema 804). All data were stored on magnetic tape throughout the entire electrophysiological study. The atrial electrograms remained constant and reproducible at each recording site in individual patients.

The bipolar electrograms were recorded from 12 sites in the right atrium in each patient. The intra-atrial catheter mapping procedure is described elsewhere. ${ }^{7}$ The high and middle atrial sites were mapped by the catheter inserted through the femoral vein and the low atrial sites were mapped through the subclavian vein. No complications were noted in any patient as a result of these manoeuvres. Stability was ensured by recording at each site for a minimum of 8-15 s and by using a looped catheter. The position of the catheter tip was verified by multiple or single plane fluoroscopy in the presence of at least two experienced physicians familiar with the right atrial endocardial mapping procedure. All the instruments were properly isolated and earthed.
Figure 1 Six atrial electrograms recorded at different right atrial sites in two patients with sick sinus node syndrome $(A$, $B$, and $C$ in a group 2 patient; $D, E$, and $F$ in a group 3 patient). Diagonal arrows represent the onset and offset of local electrical activity and horizontal arrows the downward deflections. See text for details.
A

v1

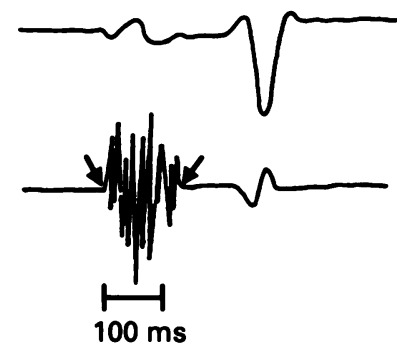

D
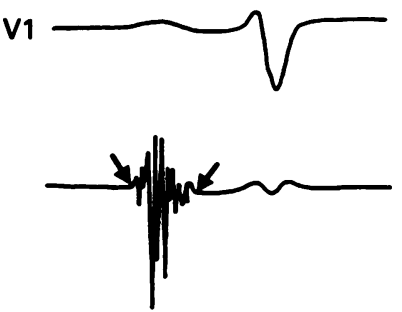
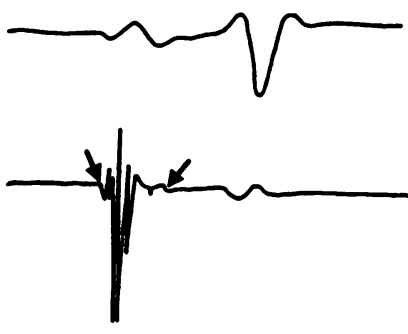

E
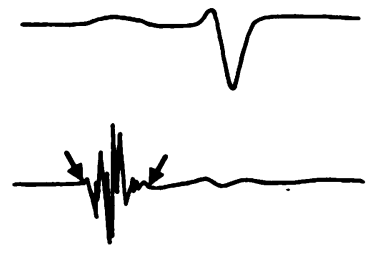
Total number (\%) of abnormal atrial electrograms in each of the three groups

Group $3 v$ group 1, p $<0.0001$; Group $3 v$ group 2, $\mathrm{p}<0.0001$; Group $2 v$ group 1, $\mathrm{p}<0.0002$

\begin{tabular}{|c|c|c|c|}
\hline & $\begin{array}{l}\text { Number of } \\
\text { electrograms }\end{array}$ & $\begin{array}{l}\text { Normal } \\
\text { electrograms (\%) }\end{array}$ & $\begin{array}{l}\text { Abnormal } \\
\text { electrograms (\%) }\end{array}$ \\
\hline $\begin{array}{l}\text { Group } 1 \\
\text { Group } 2 \\
\text { Group } 3\end{array}$ & $\begin{array}{l}479 \\
391 \\
325\end{array}$ & $\begin{array}{ll}465 & (97 \cdot 08) \\
355 & (90 \cdot 79) \\
246 & (75 \cdot 69)\end{array}$ & $\begin{array}{lr}14 & (2 \cdot 92) \\
36 & (9 \cdot 21) \\
79 & (24 \cdot 31)\end{array}$ \\
\hline
\end{tabular}

fragmented deflections. Figure $1 \mathrm{E}$ was recorded from the mid posterior right atrium and shows an abnormal atrial electrogram $105 \mathrm{~ms}$ in duration with eight fragmented deflections. Figure $1 \mathrm{~F}$ was recorded from the low posterior right atrium and shows an abnormal atrial electrogram $100 \mathrm{~ms}$ in duration with eight fragmented deflections.

\section{STATISTICAL ANALYSIS}

The duration of and the number of fragmented deflections on the atrial electrograms were assessed at each site by at least two independent observers. A minimum of five to eight atrial complexes of uniform configuration were assessed at each of the 12 sites of the atrium, and when interobserver measures did not accord a mean value was calculated. Prevalence was determined by $\chi^{2}$ test. was crossed. The number of fragmented deflections was measured by counting the number of downward deflections ${ }^{7}$ (fig 1).

In an earlier study ${ }^{7}$ we defined quantitative standards for normal bipolar electrograms. An abnormal atrial electrogram was defined as lasting $\geqslant 100 \mathrm{~ms}$ or as eight or more fragmented deflections or both.

Figure 1 shows six examples of the measurements on the atrial electrograms recorded from different right atrial endocardial sites in a patient with sick sinus syndrome but without tachycardia (A, B, and C) and in a patient with both sick sinus syndrome and paroxysmal atrial fibrillation (D, E, and F). Figure 1A was recorded from the high lateral right atrium and shows an abnormal atrial electrogram $130 \mathrm{~ms}$ in duration with $10 \mathrm{frag}$ mented deflections. Figure $1 \mathrm{~B}$ was recorded from the mid lateral right atrium and shows an abnormal atrial electrogram $110 \mathrm{~ms}$ in duration with six fragmented deflections. Figure $1 \mathrm{C}$ was recorded from the low lateral right atrium and shows a normal atrial electrogram $80 \mathrm{~ms}$ in duration with two fragmented deflections. Figure 1D was recorded from the high posterior right atrium and shows an abnormal atrial electrogram $110 \mathrm{~ms}$ in duration with nine

\begin{tabular}{|c|c|c|c|c|c|c|}
\hline & Lateral & Anterior & Posterior & Medial & & \\
\hline$\underset{R A}{\text { High }}$ & 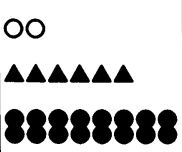 & 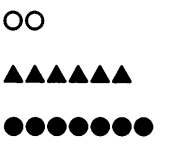 & 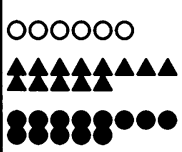 & 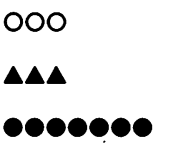 & $\begin{array}{l}13 \\
28 \\
43\end{array}$ & 84 \\
\hline $\begin{array}{l}\text { Mid } \\
\text { RA }\end{array}$ & $\begin{array}{l}\Delta \Delta \\
00000000\end{array}$ & 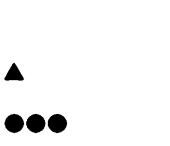 & $\begin{array}{l}\Delta \Delta \Delta \Delta \\
\bullet \bullet \bullet \bullet \bullet \bullet \bullet\end{array}$ & $\begin{array}{l}\Delta \\
\bullet 000\end{array}$ & $\begin{array}{l}0 \\
8 \\
22\end{array}$ & 30 \\
\hline $\begin{array}{l}\text { Low } \\
\text { RA }\end{array}$ & 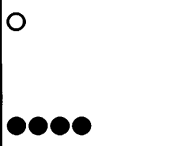 & $\bullet$ & $\bullet$ & 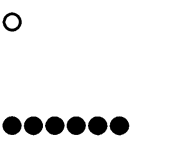 & $\begin{array}{c}2 \\
0 \\
14\end{array}$ & 16 \\
\hline & 39 & 21 & 45 & 25 & & 130 \\
\hline
\end{tabular}

O Group $1 \Delta$ Group $2 \bigcirc$ Group 3

Figure 2 Sites in the right atrium with abnormal atrial electrograms in the three groups. One symbol represents one abnormal atrial electrogram. See text for details.

\section{Results}

We assessed 1195 atrial endocardial electrograms (table) (479 in group 1, 391 in group 2 , and 325 in group 3$)$. There were $14(2.92 \%)$ abnormal atrial electrograms in group 1,36 $(9.21 \%)$ in group 2 , and $79(24.31 \%$ in group 3 . (group $3 v$ group $1, \mathrm{p}<0.0001$; group $3 v$ group 2, p $<0.0001 ;$ group $2 v$ group $1, \mathrm{p}<$ 0.0002).

SITE OF ORIGIN OF ABNORMAL ATRIAL

ELECTROGRAMS

Figure 2 shows the sites in the right atrium where abnormal electrograms were seen in the three groups. Forty three $(54 \%)$ of the 79 abnormal atrial electrograms in group 3 were seen at the high right atrium, $22(28 \%)$ at the mid right atrium, and $14(18 \%)$ at the low right atrium (high right atrium $v$ mid right atrium, $\mathrm{p}$ $<0.05$ and high right atrium $v$ low right atrium, $\mathrm{p}<0.002$ ). There was no significant

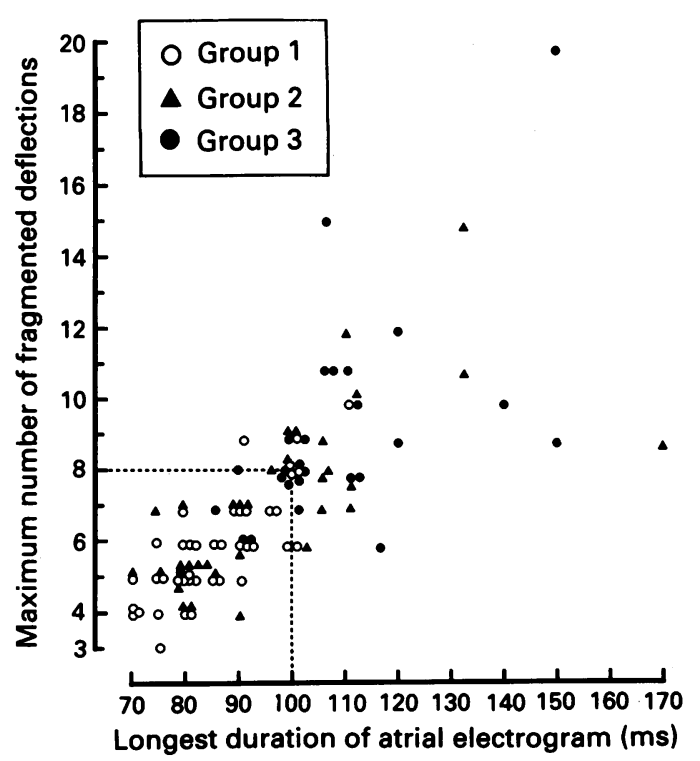

Figure 3 Longest duration and the maximal number of fragmented deflections of atrial electrograms, at 12 sites in the right atrium in each patient in the three groups. One symbol represents one patient. The broken lines indicate the limits of normality. Symbols on the lines or outside the the limits of normality. Symbols on the lines or
square formed by the lines are abnormal results. 
difference in the number of abnormal electrograms between the mid and low right atrium in group 3 patients. Of the 43 abnormal electrograms at the high right atrium, $67 \%$ were recorded from the posterolateral high right atrium. Seventy eight percent of the patients in group 3 with abnormal electrograms had them in the posterolateral high right atrium.

We recorded 36 abnormal atrial electrograms in group 2 patients; $28(78 \%)$ in the high right atrium, eight $(22 \%)$ in the mid right atrium, and none in the low right atrium (high right atrium $v$ mid right atrium, $\mathrm{p}<0.01$; mid right atrium $v$ low right atrium, $\mathrm{p}<0.02$; high right atrium $v$ low right atrium, $\mathrm{p}<0.0001$ ). Of the 28 abnormal electrograms at the high right atrium, $68 \%$ were recorded from the posterolateral high right atrium. In group 2 $88 \%$ of the patients with abnormal electrograms had them recorded in the posterolateral high right atrium.

Figure 3 shows the longest duration and the greatest number of fragmented deflections for atrial electrograms recorded at the 12 right atrial sites in each patient in the three groups.

\section{Discussion}

Fractionated electrograms were recorded from areas of canine ventricular tissue where so much fibrous tissue separated the muscle fibres from each other that the fibres were no longer arranged in parallel. ${ }^{19}$ They were also recorded from canine ${ }^{20}$ and human ${ }^{21}$ atrial muscle in which the muscle fibres were widely separated and their orientation distorted by connective tissue to such a degree that the number of intercellular connections was reduced. This could increase resistance to current flow and thereby slow conduction.

A prolonged and fractionated atrial electrogram may reflect unsynchronised local electrical activity related to a delayed and nonuniform anisotropic conduction through a diseased atrial muscle. Therefore, abnormal electrograms indicate areas of altered anatomy and conduction where reentrant arrhythmias are likely to develop.

We found a significantly greater number of abnormal endocardial electrograms in the high right atrium of patients with sick sinus syndrome irrespective of the presence or absence of paroxysmal atrial fibrillation. However, there was no significant difference between the number of abnormal electrograms recorded from the mid and low right atrium in patients with sick sinus syndrome and paroxysmal atrial fibrillation, suggesting that in these patients the electrophysiological abnormality of the atrial muscle was more diffuse. In the patients with sick sinus syndrome alone no abnormal endocardial electrograms were recorded from the low right atrium, which suggested a more localised electrophysiological abnormality.

Pathological studies showed that patients with sinoatrial block without tachycardia had disease localised to the sinus node and its approaches, whereas patients with the bradycardia-tachycardia syndrome had a more diffuse lesion of the atrium. ${ }^{22}$ Our electro- physiological studies accorded with these histological findings in that we found that abnormal atrial electrograms were generally localised to the high right atrium in the vicinity of the sinus node in patients with sick sinus syndrome without tachycardia whereas in patients with both sick sinus syndrome and paroxysmal atrial fibrillation they were more widely distributed. We believe that the greater the extent of the compromised atrial muscle, the higher the probability that paroxysmal atrial fibrillation will develop in patients with sick sinus syndrome.

The interelectrode distance of bipolar catheters, the filter frequency settings, ${ }^{23}$ the high gain recordings, and the motion at the electrode-tissue interface ${ }^{24}$ may affect the recording of fragmented electrograms by the endocardial mapping technique. We used a catheter with a $10 \mathrm{~mm}$ interelectrode distance, which is the standard catheter used by most laboratories. Catheters with such a wide interelectrode distance can record more distant electrical activity. Knowledge of the effect that filter settings have on electrogram duration is not fully complete, but the filter setting is known to influence the amplitude of the electrograms. The narrower the band pass selected, the lower the amplitude of the recorded signal obtained. We measured the duration and the number of fragmented deflections of the electrograms not their amplitude. All the atrial endocardial electrograms in our study were recorded at a fixed gain setting and they remained constant and reproducible at each recording site in individual patients.

Although these fractionated electrograms could be non-specific abnormalities or even artefacts of the recording technique, there is a well established association between their presence and the occurrence of sustained ventricular tachycardia in patients with ischaemic heart disease, ${ }^{25-27}$ that arises from areas of asynchronous and slow conducted depolarisations. Furthermore, a prolonged and fractionated atrial electrogram is known to be characteristic of paroxysmal atrial fibrillation that is closely associated with vulnerability of the atrial muscle.

Fragmented electrical activity is often recorded by a local atrial electrogram in response to single premature atrial stimulation in patients with paroxysmal atrial fibrillation. ${ }^{28-30}$ Furthermore, fragmented atrial electrograms suggesting areas of abnormal conduction have been recorded within the circuit in human atrial flutter. ${ }^{31}$ Recent clinical mapping studies during atrial flutter showed that fragmented electrograms with a double-spike pattern were recorded from the posterior and posterolateral right atrial wall in most patients. ${ }^{32} 33$ We studied the atrial electrograms during sinus rhythm by means of an endocardial catheter mapping technique. Thus we recorded fragmented double-spike electrograms in only one patient with sick sinus syndrome. This finding is consistent with that of Olshansky et al $^{33}$ who performed sequential site atrial mapping during sinus rhythm expressly to search for double potentials in nine patients. They found double 
potentials only in one patient though all their patients had double-spike electrograms during atrial flutter.

Our results help to elucidate the mechanisms responsible for precipitating paroxysmal atrial fibrillation in patients with sinus node dysfunction. We found that the prolonged and fractionated electrical activity represented anatomic areas of the atrial muscle where reentry was likely. We found that abnormal atrial electrograms were generally localised to the high right atrium in patients with sick sinus syndrome who did not have tachycardia, whereas they were more widely distributed in the right atrium of patients with both sick sinus syndrome and paroxysmal atrial fibrillation. The greater the extent of the compromised atrial muscle the higher the probability that paroxysmal atrial fibrillation will develop in patients with sick sinus syndrome.

We thank Hideko Dozaki, Naoko Sakiyama, and Yuko Sato for their excellent secretarial assistance.

1 Boineau JP, Cox JL. Slow ventricular activation in acute myocardial infarction: A source of re-entrant premature ventricular contractions. Circulation 1973;48:702-13.

2 El-Sherif N, Scherlag BJ, Lazzara R, Hope RR. Re-entrant ventricular arrhythmias in the late myocardial infarction period: 1 . Conduction characteristics in the infarction zone. Circulation 1977;55:686-702.

3 Josephson ME, Horowitz LN, Farshidi A. Continuous local electrical activity: A mechanism of recurrent ventricular tachycardia. Circulation 1978;57:659-65.

4 Wiener I, Mindich B, Pitchon R. Determinants of ventricular tachycardia in patients with ventricular aneurysms: Results of intraoperative epicardial and endocardial mapping. Circulation 1982;65:856-61.

5 Brugada P, Abdollah H, Wellens HJJ. Continuous electrical activity during sustained monomorphic ventricular tachycardia: Observations on its dynamic behavior during the arrhythmia. Am J Cardiol 1985;55:402-11.

6 Josephson ME, Wit AL. Fractionated electrical activity and continuous electrical activity: Fact or artifact? Circulation 1984;70:529-32.

7 Tanigawa M, Fukatani M, Konoe A, Isomoto S, Kadena M, Hashiba $K$. Prolonged and fractionated right atrial electrograms during sinus rhythm in patients with paroxysmal atrial fibrillation and sick sinus node syndrome. $J \mathrm{Am} \mathrm{Coll}$ Cardiol 1991;17:403-8.

8 Sutton R, Kenny R. The natural history of sick sinus syndrome. PACE 1986;9:1110-4.

9 Obel IWP, Cohen E, Millar RNS. Chronic symptomatic sinoatrial block: A review of 34 patients and their treatment. Chest 1974;65:397-402.

10 Wellens HJ, Durrer D. Wolff-Parkinson-White syndrome and atrial fibrillation: Relation between refractory period of accessory pathway and ventricular rate during atrial of accessory pathway and ventricular rate
fibrillation. Am J Cardiol 1974;34:777-82.

11 Fukatani M, Tanigawa M, Mori M, Konoe A, Kadena A Shimizu A, Hashiba K. Prediction of a fatal atrial fibrillation in patients with asymptomatic WolfParkinson-White Pattern. Jpn Circ J 1990;54:1331-9.

12 Paul T, Guccione P, Garson Jr A. Relation of syncope in young patients with Wolff-Parkinson-White syndrome to rapid ventricular response during atrial fibrillation. $\mathbf{A m}$ Cardiol 1990;65:318-21.
13 Rubenstein JJ, Schulman CL, Yurchak PM, De Sanctis $\mathrm{RW}$. Clinical spectrum of the sick sinus syndrome. Circulation 1972;46:5-13.

14 Shimizu A, Fukatani M, Kitano K, Tanigawa M, Ueyama C Kiya F, Yano K, Hashiba K. The total heart beats per 24 hours by ambulatory electrocardiography and the changes of heart rate by treadmill exercise test in sick sinus of heart rate by treadmill exercise

15 Ferrer MI. The sick sinus syndrome. Circulation 1973;47: 635- 41 .

16 Ferrer MI. The sick sinus syndrome in atrial disease. JAMA 1968;206:645-6.

17 Mandel W, Hayakawa H, Danzig R, Marcus HS. Evaluation of sino-atrial node function in man by overdrive suppression. Circulation 1971;44:59-65.

18 Narula OS, Samet P, Javier RP. Significance of the sinus node recovery time. Circulation 1972;45:140-58.

19 Gardner PI, Ursell PC, Fenoglio JJ Jr, Wit AL. Electrophysiologic and anatomic basis for fractionated electrograms recorded from healed myocardial infarcts. Circulation 1985;72:596-611.

20 Spach MS, Miller III WT, Dolber PC, Kootsey JM Sommer JR, Mosher CE, Jr. The functional role of structural complexities in the propagation of depolarization in the atrium of the dog: Cardiac conduction disturbances due to discontinuities of effective axial resistivity. Circ Res 1982;50:175-91.

21 Spach MS, Dolber PC. Relating extracellular potentials and their derivatives to anisotropic propagation at microscopic tevel in human cardiac muscle: Evidence for electrical uncoupling of side-to-side fiber connections with increasuncoupling of side-to-side fiber con

22 Thery C, Gosselin B, Lekieffre J, Warembourg H. Pathology of sinoatrial node. Correlations with electrocardiographic findings in 111 patients. Am Heart $J$ 1977;93: 735-40.

23 Waxman HL, Sung RJ. Significance of fragmented ventricular electrograms observed using intracardiac recording techniques in man. Circulation 1980;62:1349-56.

24 Ideker RE, Lofland GK, Bardy GH, Smith WM, Worley SJ, Wallace AG, Cox JL, Gallagher JJ. Late fractionated potentials and continuous electrical activity caused by electrode motion. PACE 1983;6:908-14.

25 Josephson ME, Horowitz LN. Electrophysiologic approach to therapy of recurrent sustained ventricular tachycardia. Am J Cardiol 1979;43:631-42.

26 Wiener I, Mindich B, Pitchon R. Fragmented endocardial electrical activity in patients with ventricular tachycardia: A new guide to surgical therapy. Am Heart $J 1984 ; 107$ : A6-90.

27 Klein H, Karp RB, Kouchoukos NT, Zorn GL, James TL Waldo AL. Intraoperative electrophysiologic mapping of sinus rhythm in patients with previous myocardial infarction: Identification of the electrophysiologic substrate of ventricular arrhythmias. Circulation 1982;66:847-53.

28 Hashiba K, Tanigawa M, Fukatani M, Shimizu A, Konoe A Kadena M, Mori $M$. Electrophysiologic properties of atrial muscle in paroxysmal atrial fibrillation. Am J Cardio 1989;64:20-3.

29 Ohe T, Matsuhisa M, Kamakura S, Yamada J, Sato I, Nakajima K, Shimomura K. Relation between the widening of the fragmented atrial activity zone and atrial fibrillation. Am J Cardiol 1983;53:1219-22.

30 Shimizu A, Fukatani M, Tanigawa M, Mori $M$, Hashiba $K$ Intra-atrial conduction delay and fragmented atrial activity in patients with paroxysmal atrial fibrillation. $J p n$ activity in patients with
Circ $J 1989 ; 53: 1023-30$.

31 Cosio FG, Arribas F, Palacios J, Tascon J, Lopez-Gil M Fragmented electrograms and continuous electrical Fragmented electrograms and continuous electrica

32 Cosio FG Arial futter. Am J Cardiol 1986;57:1309-14. Validation of double-spike electrograms as markers of Validation of double-spike electrograms as markers of conduction delay

33 Olshansky B, Okumura K, Henthorn RW, Waldo AL. Characterization of double potentials in human atrial flutter: Studies during transient entrainment. J Am Coll Cardiol 1990;15:833-41. 